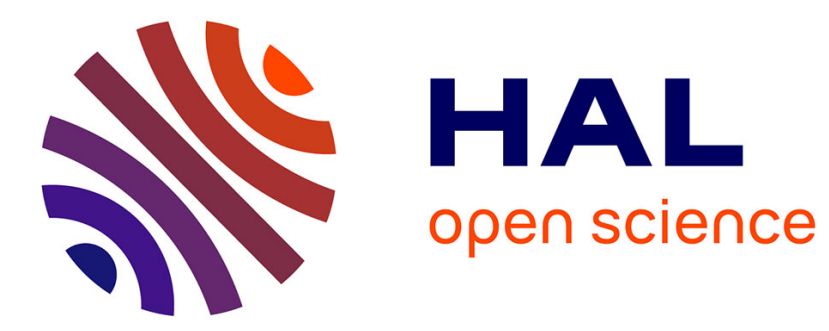

\title{
Performances d'une sonde atomique à temps de vol
}

\author{
C. Martin, D. Blavette, J.M. Sarrau
}

\section{To cite this version:}

C. Martin, D. Blavette, J.M. Sarrau. Performances d'une sonde atomique à temps de vol. Revue de Physique Appliquée, 1984, 19 (1), pp.27-31. 10.1051/rphysap:0198400190102700 . jpa-00245154

\section{HAL Id: jpa-00245154 https://hal.science/jpa-00245154}

Submitted on 1 Jan 1984

HAL is a multi-disciplinary open access archive for the deposit and dissemination of scientific research documents, whether they are published or not. The documents may come from teaching and research institutions in France or abroad, or from public or private research centers.
L'archive ouverte pluridisciplinaire HAL, est destinée au dépôt et à la diffusion de documents scientifiques de niveau recherche, publiés ou non, émanant des établissements d'enseignement et de recherche français ou étrangers, des laboratoires publics ou privés. 
Classification

Physics Abstracts

07.80

\title{
Performances d'une sonde atomique à temps de vol
}

\author{
C. Martin, D. Blavette et J. M. Sarrau \\ Groupe de Métallurgie Physique, ERA 258, UER des Sciences et des Techniques de Rouen, \\ BP 67, 76130 Mont Saint Aignan, France
}

(Reçu le 16 juin 1983, révisé le 9 septembre, accepté le 19 septembre 1983)

\begin{abstract}
Résumé. - Les performances d'une sonde atomique à temps de vol sont décrites. L'échantillon est refroidi par un cryogénérateur et le couplage de l'impulsion sur la pointe est effectué par un anneau placé à proximité. La mesure du temps de vol est faite à 2,5 ns près. Des résultats expérimentaux montrent que la résolution en masse est acceptable même pour des faibles longueurs de vol. Après avoir décrit succinctement le système d'acquisition de la sonde, un dispositif de contrôle de la tension d'évaporation est présenté.
\end{abstract}

\begin{abstract}
The performances of a time of flight atom-probe are described. The specimen is cooled with the help of a cryogenerator and the pulse coupling on the tip is achieved with a nearby ring. The time of flight measurement is made with a precision of $2.5 \mathrm{~ns}$. Experimental results show that the mass resolution is acceptable even for short flight paths. The acquisition system is briefly described and an evaporation voltage control system is submitted.
\end{abstract}

- Le microscope ionique et la sonde atomique font appel à l'émission ionique de champ où le pouvoir des pointes est utilisé pour créer un champ électrique intense (quelques dizaines de volt par $\mathrm{nm}$ ). Le principe de ces méthodes d'analyse a été décrit par ailleurs [1-3].

- L'échantillon, placé dans une enceinte à vide, est porté à un potentiel positif de plusieurs kilovolts. Lorsqu'un gaz dit " gaz image " est introduit sous faible pression, celui-ci vient s'ioniser préférentiellement au niveau des protubérances (à l'échelle atomique) de l'échantillon. L'impact sur un écran des ions formés donne alors une image agrandie de la répartition des atomes à la surface de l'échantillon.

- La sonde atomique, associée au microscope ionique permet d'identifier la nature chimique des espèces en surface. L'échantillon est évaporé atome par atome et une partie des ions métalliques formés est reçue sur un détecteur. Leur rapport masse sur nombre de charges $M / n$ est mesuré par spectrométrie à temps de vol. L'évaporation couche atomique par couche atomique de l'échantillon permet son analyse chimique en profondeur.

- La grande résolution spatiale de l'analyse tant en surface $(\sim 1 \mathrm{~nm})$ qu'en profondeur (une distance réticulaire) procure à la sonde atomique des possibilités uniques dans l'étude des alliages, notamment dans l'étude de petits précipités [4] ou encore de l'ordre à grande distance [5].

- Nous décrivons dans cet article les performances et les caractéristiques d'une sonde atomique conventionnelle. Dans ce type de sonde $\left({ }^{1}\right)$, la définition du temps de départ des ions évaporés, dont dépend la mesure du temps de vol, est obtenue en augmentant brusquement le champ électrique. Plusieurs améliorations notamment dans la mesure des temps de vol et dans le refroidissement de l'échantillon sont apportées par rapport à la sonde précédemment décrite [1].

\section{Méthode d'évaporation.}

La méthode classiquement utilisée pour évaporer les atomes d'un échantillon métallique consiste à le porter à un potentiel continu $V_{0}$ et à lui ajouter un potentiel impulsionnel $V_{\mathrm{p}}$ pour atteindre le champ d'évaporation.

Une structure coaxiale du porte échantillon peut être adoptée pour amener dans de bonnes conditions l'impulsion sur la pointe [6]. La réalisation est compliquée car difficilement compatible avec l'isolement

( $\left.{ }^{1}\right)$ L'évaporation des atomes peut aussi être obtenue par des impulsions laser qui viennent augmenter brusquement la température à la surface de l'échantillon. 
électrique nécessaire et le refroidissement de l'extrémité de la ligne porte-pointe.

Une autre méthode décrite par Panitz [7] utilise un anneau placé devant l'échantillon toujours porté au potentiel continu $V_{0}$. Une impulsion négative est appliquée sur l'anneau et l'augmentation de champ qui en résulte au niveau de la pointe provoque l'évaporation des atomes dans les mêmes conditions.

Cette disposition a l'avantage de séparer véritablement le potentiel $V_{0}$ du potentiel impulsionnel $V_{\mathrm{p}}$. L'impulsion est propagée dans une ligne coaxiale rigoureusement adaptée à son extrémité très proche de l'anneau. La structure de la tête de microscope est plus simple et est équivalente à un microscope ionique avec cependant la possibilité d'orienter l'échantillon.

La figure 1 donne le schéma de la réalisation. L'anneau est placé à $4 \mathrm{~mm}$ de la pointe et la résistance d'adaptation de la ligne est à $50 \mathrm{~mm}$ du centre de l'anneau. L'impulsion est produite par un générateur à relais (interrupteur à contact mouillé au mercure). Un condensateur est chargé par une source haute tension $V_{\mathrm{p}}$ et est déchargé dans la ligne adaptée d'impédance caractéristique $100 \Omega$ La source $V_{\mathrm{p}}$ est programmée par une tension proportionnelle à $V_{0}$. Ainsi lors de l'évolution du potentiel $V_{0}$ d'évaporation des atomes, la valeur de l'impulsion reste proportionnelle à $V_{0}$, soit $V_{\mathrm{p}}=a V_{0}$ [8]. De plus le coefficient de proportionnalité $a$ est réglable de 0,05 à 0,3 avec $V_{\mathrm{p}}<4 \mathrm{kV}$.

\section{L'orientation de l'échantillon.}

Le principe du mouvement a déjà été décrit [6]. Il combine une ouverture angulaire $\phi$ et une rotation. Des perfectionnements ont été apportés à ce mouvement, ils permettent en particulier de placer l'extrémité de l'échantillon métallique au centre du mouvement d'orientation. Il en résulte que l'alignement pointe, anneau, détecteur est maintenu quelle que soit l'orientation de la tête. Ainsi le site analysé correspond à celui choisi à l'aide de l'ensemble galette de microcanaux-écran lorsqu'il est placé en position d'observation:

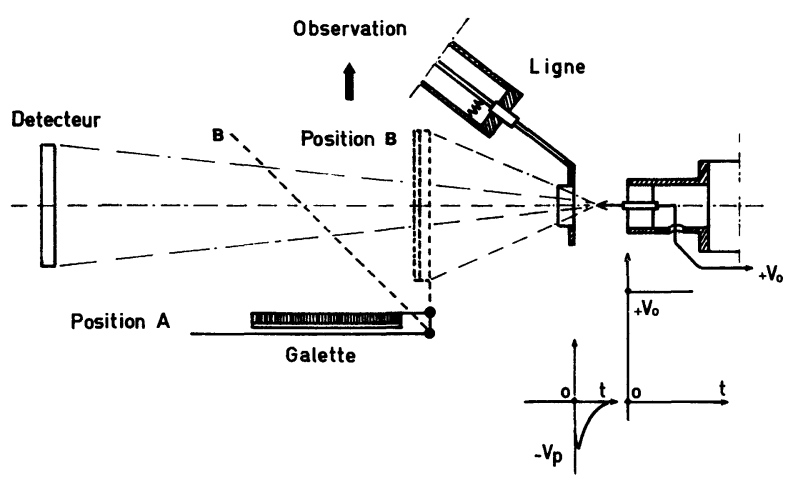

Fig. 1. - Schéma synoptique de la sonde atomique.

[Schematic diagram of the atom-probe.]
Pour obtenir facilement le réglage, il a été ajouté au système [6] un dispositif permettant de contrôler la position et le déplacement de la pointe. Les quatre vis calantes donnent les déplacements en $x, y, z$ et compensent ainsi les différentes longueurs des échantillons et les variations de longueur dues à la dilatation du porte-échantillon lors de la mise en froid (Fig. 2). Quatre hublots en regard deux à deux et montés à $90^{\circ}$ ont leurs axes respectifs passant par le centre de rotation $\mathrm{C}$. Ainsi le réglage se fait en observant directement à travers un hublot l'extrémité de la pointe à l'aide d'une lunette de visée. L'extrémité de l'échantillon est amenée en $C$ avec une imprécision de $0,1 \mathrm{~mm}$.

Lors d'un changement de site d'analyse, c'est-àdire pour de nouvelles valeurs de l'ouverture angulaire et de la rotation, la position de l'apex reste inchangée. Le site d'analyse est ainsi parfaitement déterminé.

\section{Le refroidissement de l'échantillon.}

Deux systèmes sont utilisés pour refroidir l'échantillon. Le premier dispositif déjà utilisé [6] comprend un petit cryostat qui est rempli soit d'azote liquide, soit d'hélium liquide. La tête du microscope est refroidie par la circulation du fluide réfrigérant qui est entretenue par une dépression obtenue par une pompe mécanique.

Le second dispositif utilise un cryogénérateur (LEYBOLD HERAEUS SOGEV RG 210) fixé directement sur le support de la tête de microscope, il est orien a e avec ec an 1 on. po e-ec anti on est raccordé au cryogénérateur par une pièce cylindrique en cuivre percée de quatre trous. Quatre fils sont disposés suivant l'axe des trous pour constituer un ensemble de résistances de chauffage. En faisant passer un courant dans ces fils, il est possible de régler la température du porte-échantillon et de le réchauffer rapidement lors d'un changement de spécimen. La température obtenue avec ce système est inférieure à $20 \mathrm{~K}$.

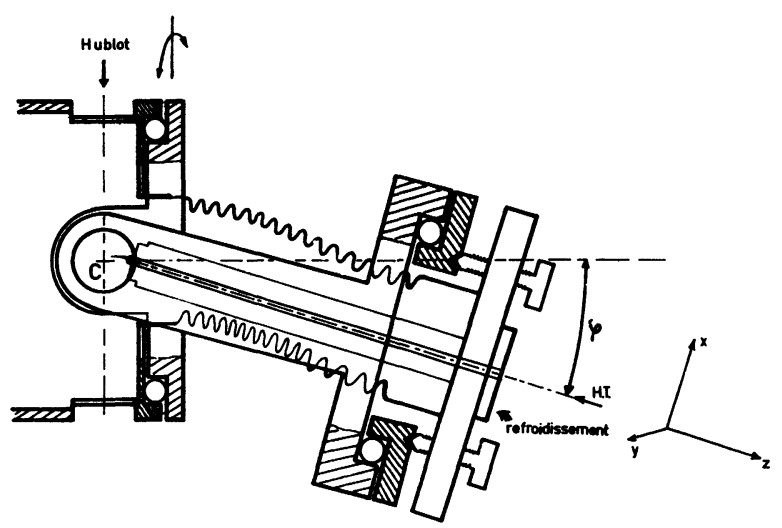

Fig. 2. - Schéma de la tête goniométrique.

[Scheme of the goniometer head.] 


\section{La mesure du temps de vol.}

La mesure du temps de vol des ions est assurée par une chaîne de comptage dont le principe de fonctionnement est semblable à celui décrit précédemment [1]. Cette chaîne est constituée de trois compteurs dont l'ouverture est commandée par l'impulsion d'évaporation, et d'un registre à décalage assurant leur fermeture sur l'arrivée successive des ions évaporés. Le système est élaboré en technologie ECL. En particulier, le registre à décalage est constitué d'un circuit logique MOTOROLA 1964 de la série ECL III et les décades de tête $(700 \mathrm{MHz})$ sont des circuits PLESSEY 8634.

La fréquence de récurrence de l'horloge pilote est portée à $400 \mathrm{MHz}$ ce qui autorise des longueurs de vol plus faibles sans perte notable de résolution en masse. La figure 3 montre le spectre d'un super alliage à base nickel pour une longueur de vol de $0,90 \mathrm{~m}$. La figure 4 permet de comparer les résultats obtenus pour des fréquẹnces horloge de $200 \mathrm{MHz}$ et $400 \mathrm{MHz}$, et une longueur de vol $\left(l_{v}\right)$ de $33 \mathrm{~cm}$. Seuls les spectres obtenus à $400 \mathrm{MHz}$ permettent de séparer les deux isotopes de l'iridium avec une résolution en masse de l'ordre de $7 / 1000$.

Il faut noter dans ce cas que les différences de longueur de vol entre le centre et la périphérie du détecteur plan introduisent une erreur perceptible pour une telle distance de vol.

Le diamètre $\phi$ du détecteur étant de $4 \mathrm{~cm}$ l'incertitude introduite sur la masse est : $\frac{\Delta m}{m}=\frac{\phi^{2}}{8 l_{\mathrm{v}}^{2}} \cong \frac{2}{1000}$.

\section{Le système d'acquisition.}

L'expérience est gérée par un séquenceur et un microordinateur EXORSET 30 (MOTOROLA) (Fig. 5). Tous les signaux de contrôle (initialisation de la chaîne de comptage, ordre d'envoi d'une impulsion, etc...) sont issus du séquenceur sous le contrôle du calculateur.

L'ensemble des données expérimentales (temps de vol, potentiel d'évaporation, nombre d'impulsions) est centralisé au niveau d'un sérialisateur. Celui-ci transmet les données à l'ordinateur à une vitesse de 2400 bauds qui en assure le traitement en ligne et le stockage sur disque.

Une portion de mémoire joue le rôle de tampon d'entrée et stocke jusqu'à 30 messages, ce système rend donc transparent les calculs et la formation des spectres en temps réel. Dans des conditions normales d'utilisation (20 impulsions/s) trois mille résultats peuvent être saisis en une heure. La diminution du temps d'acquisition va se traduire par une réduction de l'évaporation préférentielle. En effet le nombre d'atomes s'évaporant au potentiel continu (donc non détectés) est proportionnel au temps moyen $t_{\mathrm{m}}$ séparant l'arrivée de chaque ion. Si $p$ est le nombre d'impulsions séparant en moyenne la réception de chaque ion, $f$ la fréquence nominale d'envoi des impulsions

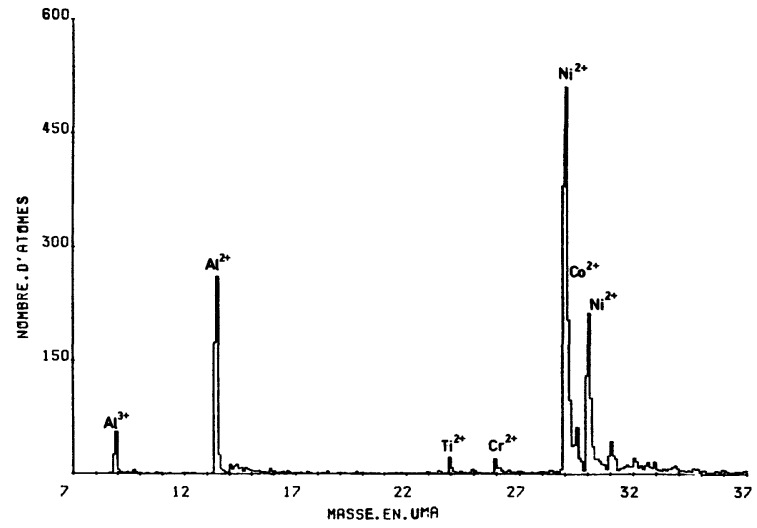

Fig. 3. - Spectre de masse d'un super alliage à base nickel. La longueur de vol est de $0,9 \mathrm{~m}$ et la résolution en temps est de $2,5 \mathrm{~ns}$.

[Mass spectrum of a nickel-based superalloy. The flight path is $0.9 \mathrm{~m}$ and the time resolution is $2.5 \mathrm{~ns}$.]
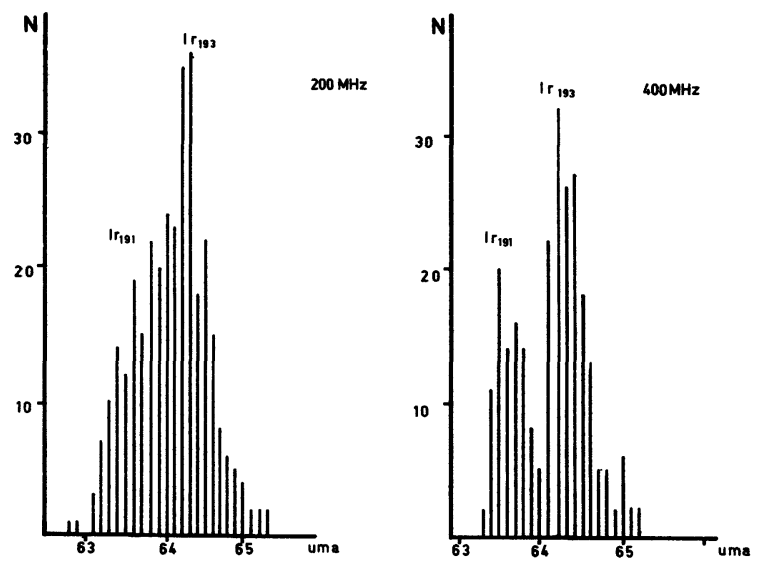

Fig. 4. - Spectre de masse d'iridium pour les deux fréquences horloge. La longueur de vol est de $33 \mathrm{~cm}$.

[Mass spectrum of iridium for two various clock frequencies. The flight path is $33 \mathrm{~cm}$.]

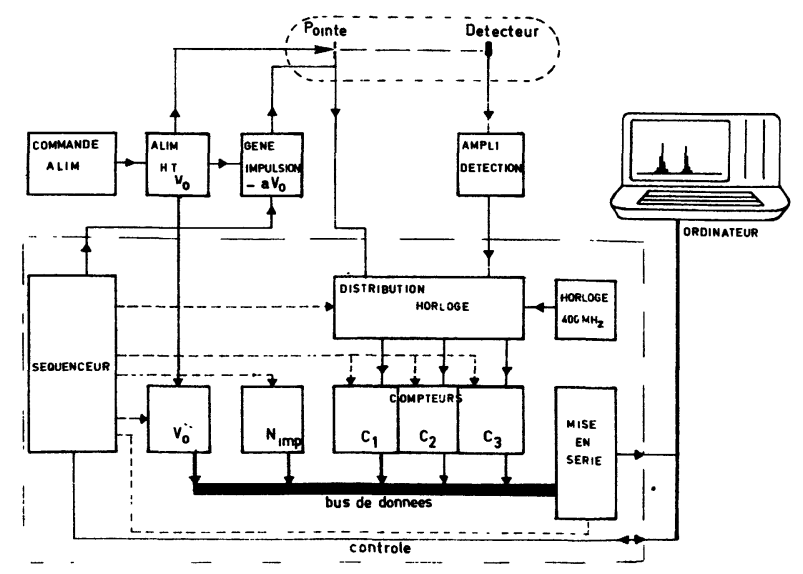

Fig. 5. - Système d'exploitation de la sonde atomique.

[Exploitation system of the atom-probe.] 
et $t_{\mathrm{a}}$ le temps d'acquisition d'un ion, $t_{\mathrm{m}}$ peut s'écrire : $t_{\mathrm{m}}=t_{\mathrm{a}}+p / f$.

Cette expression montre que pour minimiser l'évaporation préférentielle, $t_{\mathrm{a}}$ et $p$ doivent être choisis les plus faibles possibles. Il existe cependant un compromis à faire dans le choix de $p$ de manière à ce que deux ions de même masse n'arrivent pas en même temps sur le détecteur. $p$ est généralement maintenu à une valeur supérieure à $20 \mathrm{imp} / \mathrm{at}$.

\section{Contrôle de la tension continue d'évaporation.}

Le potentiel continu appliqué à l'échantillon est élaboré à partir d'une alimentation H.T. contrôlable (BERTAN $-205 \mathrm{~A}-20$ P).

Une commande de cette alimentation a été conçue de manière à assurer une montée lente et régulière du potentiel appliqué à la pointe. L'augmentation du rayon de courbure de l'échantillon (de forme conique) au fur et à mesure de son évaporation est ainsi compensée. Le champ électrique peut être ainsi maintenu sensiblement constant.

La commande est réalisée à partir d'un convertisseur digital-analogique (16 bits) et de 16 compteursdécompteurs binaires synchrones. La vitesse de montée est contrôlée par la fréquence de l'horloge pilote du système. Une avance rapide permet en outre de montrer ou descendre le potentiel manuellement. En mode automatique la vitesse de montée du potentiel d'évaporation peut être réglée de $0,18 \mathrm{~V} / \mathrm{min}$. à $18 \mathrm{~V} / \mathrm{min}$.

insi a vi esse evapora ion e ec an 1 on est finement contrôlée. Comme il a été montré $[9,10]$, le nombre de plans $(h k l)$ évaporés par unité de temps $(\mathrm{d} n / \mathrm{d} t)$ est relié à la vitesse de montée du potentiel d'évaporation $(\mathrm{d} V / \mathrm{d} t)$ par l'expression :

$$
\frac{\mathrm{d} n}{\mathrm{~d} t}=\frac{\cos (\theta+\phi) \cdot(1+a)}{E \beta \cdot k_{\alpha} \cdot k_{\theta} \cdot d_{h k l}} \frac{\mathrm{d} V}{\mathrm{~d} t},
$$

$a$ est la fraction d'impulsion;

$\theta, \phi$ et $k_{\theta}$ sont des paramètres liés au site d'analyse et $E \beta, k_{\alpha}$ des facteurs caractéristiques de l'échantillon; $d_{h k l}$ est la distance réticulaire.

La figure 6 illustre la linéarité existant entre le nombre de plans évaporés (c'est-à-dire la profondeur d'analyse) et le potentiel d'évaporation. L'exemple du plan (031) du W montre en l'occurrence que cette linéarité n'est obtenue que lorsque les conditions de champ sont devenues stationnaires. La pente de la courbe permet d'étalonner l'échelle des distances à la sonde ainsi que d'évaluer la résolution spatiale en surface [10].

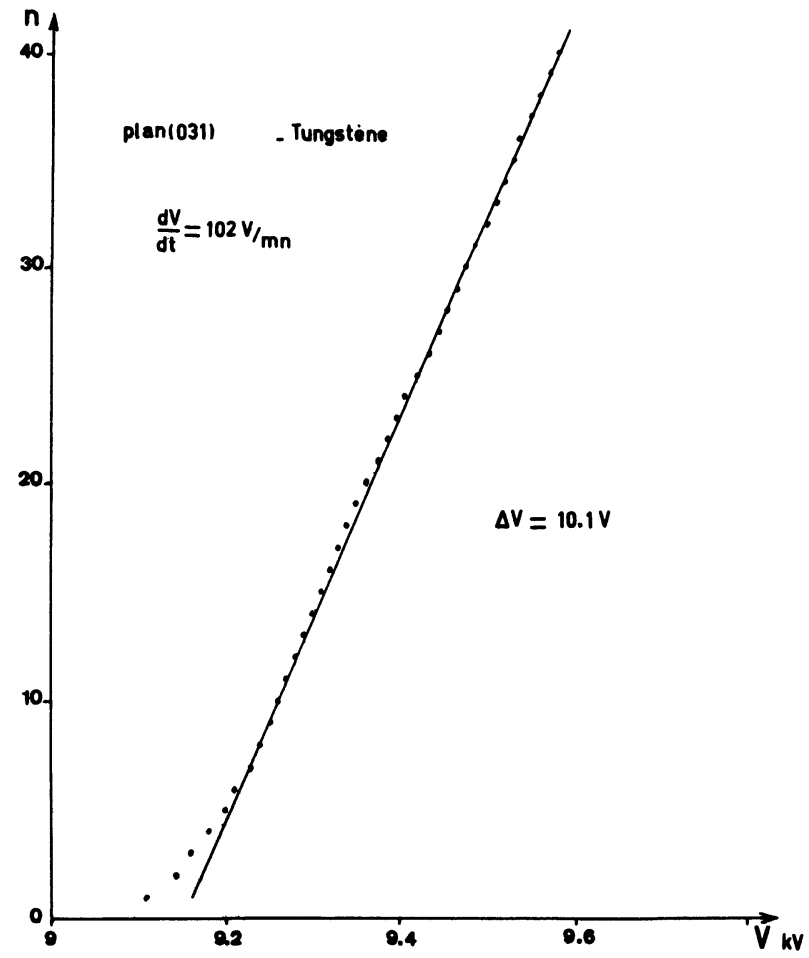

Fig. 6. - Courbe expérimentale du nombre de plans évaporés en fonction de la tension d'évaporation.

[Experimental curve of the number of evaporated planes versus the evaporation voltage.]

\section{Conclusion.}

Les améliorations apportées à la sonde atomique facilitent son utilisation dans l'étude des alliages et élargissent notablement son champ d'application. En particulier l'accès aux basses températures (cryogénérateur), ouvre la voie à l'analyse des alliages légers $(\mathrm{AlZn})$. La versatilité de la sonde en est donc accrue. La plus grande résolution en temps de vol permet d'autre part l'utilisation de la sonde en temps de vol court ce qui autorise des analyses plus globales.

La complète autonomie de l'appareil est maintenant assurée grâce à l'emploi d'un cryogénérateur (l'autonomie des cryostats utilisés était de l'ordre de $8 \mathrm{~h}$ ). Parallèlement à cela, les progrès accomplis dans le transfert et le traitement des données a permis d'augmenter considérablement la vitesse d'acquisition et donc d'accroître le rendement de la sonde atomique. Les phénomènes d'évaporation préférentielle sont de plus minimisés. 


\section{Bibliographie}

[1] Gallot, J., Sarrau, J. M., Bostel, A., Le Vide 179 (1975) 173.

[2] Blavette, D., Bostel, A., Sarrau, J. M., Revue Phys. Appl. 16 (1981) 405.

[3] Muller, E. W., Panitz, T. A., Maclane, S. B., Rev. Sci. Instrum. 39 (1968) 83.

[4] Blavette, D., Martin, C., Gallot, J., Scr. Metall. 16 (1982) 59.

[5] Blavette, D., Bostel, A., Menand, A., 30th IFES, Philadelphie (1983)
[6] Sarrau, J. M., Gallot, J., Avenel, O., Roubeau, P., J. Phys. Sci. Instrum. 14 (1981) 800.

[7] Panitz, J. A., Rev. Sci. Instrum. 44 (1973) 1034.

[8] Hall, T., Wagner, A., Seidman, D. N., Cornell Univers. Material Science Center, Report 2357 (1975).

[9] Blavette, D., Sarrau, J. M., Bostel, A., Gallot, J., Revue Phys. Appl. 17 (1982) 435.

[10] Blavette, D., Bostel, A., Sarrau, J. M., Gallot, J., Proceedings, 29th IFES (1982) 497. 